University of Nebraska - Lincoln

DigitalCommons@University of Nebraska - Lincoln

Faculty Publications, Department of Psychology

Psychology, Department of

2014

Child Advocacy Center Based Group Treatment for Child Sexual Abuse

\author{
Grace S. Hubel \\ University of Nebraska-Lincoln, hubel@musc.edu \\ Christopher Campbell \\ University of Oklahoma Health Sciences Center \\ Tiffany West \\ University of Nebraska-Lincoln \\ Samantha Friedenberg \\ University of Nebraska-Lincoln \\ Alayna Schreier \\ University of Nebraska-Lincoln
}

See next page for additional authors

Follow this and additional works at: https://digitalcommons.unl.edu/psychfacpub

Part of the Psychology Commons

Hubel, Grace S.; Campbell, Christopher; West, Tiffany; Friedenberg, Samantha; Schreier, Alayna; Flood, Mary Fran; and Hansen, David J., "Child Advocacy Center Based Group Treatment for Child Sexual Abuse" (2014). Faculty Publications, Department of Psychology. 1034.

https://digitalcommons.unl.edu/psychfacpub/1034

This Article is brought to you for free and open access by the Psychology, Department of at DigitalCommons@University of Nebraska - Lincoln. It has been accepted for inclusion in Faculty Publications, Department of Psychology by an authorized administrator of DigitalCommons@University of Nebraska - Lincoln. 


\section{Authors}

Grace S. Hubel, Christopher Campbell, Tiffany West, Samantha Friedenberg, Alayna Schreier, Mary Fran Flood, and David J. Hansen 


\title{
Child Advocacy Center Based Group Treatment for Child Sexual Abuse
}

\author{
Grace S. Hubel, ${ }^{1}$ Christopher Campbell, ${ }^{2}$ Tiffany West, ${ }^{3}$ \\ Samantha Friedenberg, ${ }^{3}$ Alayna Schreier, ${ }^{3}$ Mary Fran Flood, ${ }^{3}$ \\ and David J. Hansen ${ }^{3}$
}

1. University of Nebraska-Lincoln, Lincoln, Nebraska, USA, and Medical University of South Carolina, Charleston, South Carolina, USA

2. University of Oklahoma Health Sciences Center, Oklahoma City, Oklahoma, USA

3. University of Nebraska-Lincoln, Lincoln, Nebraska, USA

Corresponding author - Grace S. Hubel, Department of Psychiatry and Behavioral Sciences, National Crime Victims Research and Treatment Center, Medical University of South Carolina, 67 President St., MSC 861, 2nd Floor, IOP South Building, Charleston, SC 29425, email hubel@musc.edu

\begin{abstract}
The present study examines initial symptom presentation among participants, outcomes, and social validity for a group treatment for child sexual abuse delivered at a child advocacy center. Participants were 97 children and their nonoffending caregivers who were referred to Project SAFE (Sexual Abuse Family Education), a standardized, 12-week cognitive-behavioral group treatment for families who have experienced child sexual abuse. Sixty-four percent of children presented with clinically significant symptoms on at least one measure with established clinical cutoffs. Caregivers of children who presented with clinically significant symptoms reported more distress about their competence as caregivers. Children who presented as subclinical were more likely to have experienced intrafamilial sexual abuse. Posttreatment results indicated significant improvements in functioning for all children who participated in treatment, with greater improvements reported for children who initially presented with clinically significant symptoms. Overall, the program was rated favorably on the posttreatment evaluation of social validity.
\end{abstract}

Keywords: intervention, child maltreatment, community-based 
The complex nature of child sexual abuse (CSA) presents challenges for clinicians and researchers who develop and implement treatments for victims and their families. Sexually abused children are a heterogeneous group, and victimization does not necessarily result in a unified presentation of symptoms (Saywitz, Mannarino, Berliner, \& Cohen, 2000). In fact, some individuals exhibit little to no symptoms following abuse (Putnam, 2003; Wolfe, 2006). Results across studies suggest that a variety of factors may impact outcomes of CSA, including caregiver support following disclosure, family functioning (e.g., marital conflict, finances), environmental stressors, distorted cognitions, negative attributional style (by the victim or parents), problematic coping strategies, and additional forms of maltreatment (Tyler, 2002; Wolfe, 2006).

Given the heterogeneous effects of CSA, it is important that treatments address a broad range of symptom profiles (Saywitz, et al., 2000). Some children will present with serious psychiatric symptoms. Research examining outcomes for sexually abused children has demonstrated that psychological treatment can be helpful in decreasing these symptoms (Harvey \& Taylor, 2010). In other cases, children will exhibit subclinical difficulties. These children may be at risk for what other researchers have observed and called a "sleeper effect," in which symptoms emerge following a period of undetected impairment (Saywitz et al., 2000). For these children, treatment may help prevent symptoms (Saunders, Berliner, \& Hanson, 2004). Furthermore, even when children are not affected by abuse in measurable ways, treatment can prevent revictimization, promote healthy coping, and educate caregivers (Putnam, 2003).

Information on how to best treat the wide variety of difficulties families may experience following CSA is especially important for clinicians working in community settings, particularly agencies that specialize in child maltreatment such as child advocacy centers (CACs). CACs are child-friendly facilities staffed by trained professionals who use a multidisciplinary approach to conduct forensic interviews and medical examinations in addition to providing victim support and advocacy, mental health services, and community training and prevention activities (Cross, Jones, Walsh, Simone, \& Kolko, 2007). The CAC approach to serving families arose out of complaints in the 1980s that the process children were submitted to following abuse (e.g., multiple interviews with various agencies) was causing further harm (Jackson, 2004). Currently, 700 CACs exist nationally and serve as a primary resource for families after sexual abuse is disclosed (National Children's Alliance, 2009). Although the CAC model initially provided a single site to gather forensic information following abuse, CACs have expanded into multicomponent resources that recognize the mental health treatment needs of families served (Jackson, 2004; Tavkar \& Hansen, 2011). Thus, mental health professionals employed by CACs are in the position of serving a population seeking immediate services after a stressful life event rather than the emergence of symptoms of a psychological disorder.

Despite recognition of variation in postabuse functioning and the possible benefits of providing treatment to asymptomatic children, there is currently a lack of research employing samples of sexually abused children with heterogeneous symptom presentations. In fact, research on the effectiveness of CSA treatment programs can be challenging due to difficulty detecting positive change on outcome measures for symptomatically diverse children (Finkelhor \& Berliner, 1995). Furthermore, many CSA treatment outcomes studies 
focus on amelioration of symptoms that constitute a specific psychological diagnosis (Harvey \& Taylor, 2010). Despite these difficulties, it seems likely that clinicians working with families who have experienced CSA, especially those employed in CAC settings, will continue to encounter a diverse population that seeks treatment because of disclosure of abuse and not necessarily because of clinically significant symptoms of a particular disorder. Therefore, research that explores variation in children's reactions to abuse and on treatment capable of addressing these varied reactions is critical.

The current study evaluates a group treatment program for families participating in mental health services delivered at a Midwest CAC following children's disclosure of CSA. The intervention, Project SAFE (Sexual Abuse Family Education), is a 12 session, parallel (children and nonoffending caregiver groups meet separately but concurrently), manualized, cognitive behavioral group treatment for children who have experienced CSA and their nonoffending caregivers. After the establishment of a local CAC in 1998, the need for prompt, onsite mental health services was evident. Project SAFE was developed at the University of Nebraska-Lincoln and began delivering onsite mental health services at the CAC in 2000 (Tavkar \& Hansen, 2011). Project SAFE evaluations comparing children's scores on assessment measures delivered prior to and following treatment have documented improvements for children, including lower anxiety, fewer posttraumatic stress symptoms, increased basic sexual knowledge, increased self-esteem, and fewer maladaptive abuse attributions (Hubel, Maldonado, Tavkar, Hansen, \& Flood, 2011; Hsu, 2003; Sawyer et al., 2005).

Development of the Project SAFE program was based on systematic review of the literature on treatment for CSA, which revealed a need for treatments capable of addressing a heterogeneous range of symptom presentations (Hansen, Hecht, \& Futa, 1998). Treatment is provided to groups of children with varied levels and types of symptoms simultaneously. Group treatment allows children and caregivers to learn that other families have also experienced abuse and related symptoms, which may help to reduce feelings of isolation and stigmatization (Hetzel-Riggin, Brausch, \& Montgomery, 2007). The group setting also allows for opportunities to enhance social skills, practice new behaviors with peers (e.g., assertiveness), and participate in role-play situations with other group members (De Luca, Boyes, Furer, Grayston, \& Hiebert-Murphy, 1992). In addition, group treatment is a cost-effective modality, allowing multiple children with varying symptom presentations to be treated simultaneously. Project SAFE treatment focuses on three broad areas often affected by CSA: (a) the individual/self (e.g., self-esteem, self-blame, internalizing difficulties), (b) interpersonal relationships (e.g., social skills, externalizing problems with peers and family), and (c) sexual development and behaviors (e.g., sexual knowledge, sexual abuse-specific psychoeducation, sexual behavior problems). The broad focus and inclusive nature of the protocol differentiates it from individual treatment options for children who have experienced CSA, such as trauma-focused cognitive behavioral therapy (Cohen, Mannarino, \& Deblinger, 2006). Individual treatment options for CSA have typically been evaluated in research focusing on ameliorating specific trauma-related psychiatric diagnoses such as posttraumatic stress disorder (PTSD; Lang, Ford, \& Fitzgerald, 2010).

The current study examines factors potentially related to initial symptom presentation of children participating in Project SAFE, including family demographics, characteristics 
of the abuse, and pretreatment family functioning. It was expected that children who experienced more severe abuse and whose caregivers reported lower family functioning at treatment onset would be more likely to exhibit clinical levels of symptoms. This study also compares treatment outcome results for children who initially presented with clinically significant symptoms and children who initially exhibited subclinical symptomatology. The purpose of this evaluation is to provide insight into the potentially differential benefits of treatment for sexually abused children presenting with and without high levels of psychiatric symptoms. Improvement was expected across both groups, with the initially symptomatic youth showing the greatest improvement. It was also hypothesized that all families (regardless of initial symptomatology) would report satisfaction with treatment goals, content/format, and outcomes of Project SAFE.

\section{Method}

\section{Participants}

Participants were 97 children and their nonoffending caregivers who were referred to the Project SAFE group treatment program. The average group of child participants consisted of 3.00 members $(S D=1.97)$ and the average group of nonoffending caregivers consisted of 4.25 members $(S D=2.28)$. Caregiver groups were sometimes larger than child groups because multiple caregivers from the same family participated in treatment. While efforts to recruit participants aimed to create large groups, smaller groups were sometimes conducted with the goal of continually meeting the needs of the population served by the CAC. In total, 28 different groups were conducted.

Professionals at a variety of agencies, including the local CAC, the Department of Health and Human Services, and private practices serving children and families referred participants for services. Twenty-three percent of the children $(n=22)$ were male, and children ranged in age from 6.97 to 12.83 years $(M=10.00, S D=1.63)$. The average age range within groups was 2.24 years $(S D=1.69)$. Eighty children $(80 \%)$ identified as European American, 6 children (6\%) were African American, 5 children (5\%) were Hispanic/Latino, 7 (7\%) were bi- or multi-racial, and 1 child $(1 \%)$ was American Indian. Nonoffending caregivers ranged in age from 23 to 55 years $(M=35.55, S D=6.63)$. The majority of the caregivers $(n=78,80 \%)$ were the biological mother of the child, $10(10 \%)$ were the biological father, and the remaining caregivers were step or adoptive mothers, foster mothers, and grandmothers. Eighty-seven percent of the nonoffending caregivers identified as European American, $6 \%$ were Hispanic/Latino, 5\% were bi- or multi-racial, and 1\% identified as American Indian.

Thirty-seven (38\%) of nonoffending caregivers were married, 32 (33\%) were divorced, 15 (16\%) were separated, $6(6.2 \%)$ were cohabitating, 4 (4\%) were single, and $3(3 \%)$ nonoffending caregiver's marital status was unknown. The highest education level obtained for the nonoffending caregivers was distributed in the following manner: $11(11 \%)$ had not graduated from high school, 41 (42\%) were high school graduates, $20(21 \%)$ had completed some college, $9(9 \%)$ had an associate's degree, 9 (9\%) had a bachelor's degree, $4(4 \%)$ had a master's degree, and $3(3 \%)$ had an education level that was unknown. Of the 97 caregivers, $64(66 \%)$ were currently employed. Distribution of annual household income was as 
follows: $37 \%$ earned $\$ 15,000$ or less; $12 \%$ earned $\$ 15,001$ to $\$ 25,000 ; 18 \%$ earned $\$ 25,001$ to $\$ 40,000 ; 17 \%$ earned $\$ 40,001$ to $\$ 60,000 ; 10 \%$ earned $\$ 60,001$ to $\$ 100,000 ; 2 \%$ earned more than $\$ 100,000$; and $4 \%$ of the caregivers did not report their annual income.

\section{Child Measures}

Children participating in the current study were administered the following instruments at the CAC prior to the first session of treatment (pretreatment) and following the final session of treatment (posttreatment). Graduate students were available to assist children with reading instruments and recording verbal answers when necessary. Children typically took between 1 to 2 hours to complete the battery of instruments.

The Children's Depression Inventory (CDI)

The CDI (Kovacs, 1992) is a widely used self-report measure of depression for children ages 7 to 17 years. The measure includes 27 items that ask children to endorse various symptoms of depression. Instructions ask children to rate how they felt in the past 2 weeks based on 3 choices that are keyed from 0 to 2 with higher scores indicating higher symptom severity. When used in general screening, T-scores greater than 65 appear to be an appropriate cut-point indicating concern regarding symptoms of depression. The CDI has been found to be reliable with adequate internal consistency ranging from .71 to .89 and established test-retest reliability (.72 to .84; Kovacs, 1992). For the current sample, Cronbach's alpha for the CDI was .90 .

\section{Revised Children's Manifest Anxiety Scale (CMAS-R)}

The CMAS-R (Reynolds \& Richmond, 1985) assesses general symptoms of anxiety in youth ages 6 to 19 years. The measure utilizes 37 self-report items that ask children to respond by circling either yes or no. Three subscale scores can be derived, which assess Physiological Anxiety, Worry/Oversensitivity, and Social Concerns/Concentration. Total anxiety scores are based on 28 items that question about physiological, subjective, and motor symptoms of anxiety. T-scores greater than 60 suggest the need for further information, consultation, and follow-up regarding children's symptoms of anxiety. There is also a Lie Scale, which measures a child's defensiveness or inability to understand the questions. The CMAS-R has good internal consistency with an alpha of 83 for the Total Anxiety Scale (Reynolds \& Richmond, 1985). An alpha of .79 was found for the current sample. The validity and stability of this measure has also been established (Reynolds, 1980; Reynolds \& Richmond, 1985).

\section{Children's Loneliness Questionnaire (CLQ)}

The CLQ (Asher \& Wheeler, 1985) is a self-report questionnaire that assesses children's feelings of loneliness, social adequacy, and subjective estimations of peer status. The 24-item questionnaire was originally designed for use with a population of 3rd and 6th grade children (Asher \& Wheeler, 1985); however, it has since been used with youth up to age 18 years (e.g., Prinstein \& La Greca, 2002). Children are asked to rate statements on the measure using a 5-point scale ranging from that's always true about me to that's not true at all about $m e$. The CLQ has good internal consistency with an alpha of .90 for the 16 primary items 
(Asher \& Wheeler, 1985). For the current sample, alpha was .88. The validity of the CLQ distinguishing social status of children has also been established (Asher \& Wheeler, 1985).

Children's Impact of Traumatic Events-Revised (CITES-R)

The CITES-R (Wolfe, Gentile, Michienzi, Sas, \& Wolfe, 1991) is a structured interview that measures how sexual abuse has impacted children aged 8 to 16 from their own perspective (e.g., thoughts and feelings about what happened to them). The measure assesses the impact of traumatic events across areas of posttraumatic stress, abuse attributions, social reactions, and eroticism. Moderate support has been demonstrated for the psychometric properties of the CITES-R PTSD scale, including reliability with alpha ranging from .56 to .79 (Chaffin \& Shultz, 2001). Alphas for the scales utilized in the current study sample ranged from .69 to .75 .

\section{Children's Fears Related to Victimization (CFRV)}

The CFRV (Wolfe \& Wolfe, 1986), a 27-item subscale of the Fear Survey Schedule for Children Revised (FSSC-R; Ollendick, 1983; Wolfe \& Wolfe, 1986), is a self-report measure for children aged 7 to 12 years that assesses situations that may be distressing to sexually abused children (e.g., people knowing bad things about me, sleeping alone, saying "no" to an adult). This measure utilizes a 3-point scale for children to rate their level of fear in these situations. Scores range from 27 to 81, with higher scores indicating greater level of fear. The CFRV consists of two subscales: sex-associated fears and interpersonal discomfort. Both have been found to have high internal reliability, though their validity has not yet been established (Feindler, Rathus, \& Silver, 2003). A reliability coefficient of $\alpha=.85$ was found for the current sample.

\section{Child Project Safe Evaluation Form}

The Child Project SAFE Evaluation Form is a 6-item Likert scale type instrument used to ask child participants to rate their satisfaction with their treatment experience. It was specifically constructed for assessing client satisfaction with the Project SAFE program; therefore, no reliability or validity data are currently available. Table 1 contains a description of the items covered by the measure. The Child Project SAFE Evaluation Form is administered only during the posttreatment assessment. 
Table 1. Mean and Standard Deviations of Items on the Child Posttreatment Evaluation Form

\begin{tabular}{lcc}
\hline & $\begin{array}{c}\text { Symptomatic Children } \\
(n=36)\end{array}$ & $\begin{array}{c}\text { Subclinical Children } \\
(n=26)\end{array}$ \\
\cline { 2 - 3 } Item & $M(S D)$ & $M(S D)$ \\
\hline Group Therapists: & $2.72(.61)$ & $2.97(.19)$ \\
$\quad$ Warm and Understanding Toward Me & $2.92(.28)$ & $2.81(.57)$ \\
$\quad$ Knew What They Were Talking About & & \\
Group Topics: & $2.81(.47)$ & $2.65(.56)$ \\
$\quad$ Were Important to Me & $2.72(.51)$ & $2.65(.56)$ \\
Able to Understand Discussions & & \\
Overall Evaluation of Project SAFE: & $2.83(.45)$ & $2.92(.27)$ \\
$\quad$ Liked Coming to Group & $2.67(.53)$ & $2.77(.51)$ \\
Feel Like I Am Better Off Now Than When Group Began & & \\
\hline
\end{tabular}

Note: Child Project SAFE Evaluation Form items were rated on a scale of 1 (almost never) to 3 (most of the time).

\section{Caregiver Measures}

Adult participants in the current study completed the following instruments at the CAC during a pre and posttreatment assessment. Adults typically took between 1 to 1.5 hours to complete the battery of instruments.

\section{The Child Behavior Checklist (CBCL)}

The CBCL (Achenbach, 1991) is a 113-item checklist used to assess parents' perceptions of social competence and behavioral problems in their children aged 4 to 18 . This measure utilizes a 3-point scale ranging from 0 (not true) to 3 (very true or often true) for parents to rate the presence of problem behaviors during the previous 7 months. T-scores greater than 60 are in the borderline or clinical range and indicate possible behavioral problems of clinical significance. Strong evidence for the psychometric properties of the CBCL has been well established through multiple studies conducted over the past 20 years (Achenbach \& Rescorla, 2000). For the current sample, alphas for the Internalizing and Externalizing Scales were .77 and .85 , respectively.

Family Adaptability and Cohesion Evaluation Scale-III (FACES-III)

The FACES-III (Olson, 1986) is a 20-item measure used to assess family adaptability, cohesion, and satisfaction. Each item in the measure is administered twice, and respondents are asked to describe current and ideal family adaptability, cohesion, and satisfaction. The measure assesses perceptions of current and ideal family systems using a 5-point scale. The FACES-III has fair internal consistency (alphas ranging from .62 to .77) as well as good face validity (Olson, 1986). For the current sample, alpha was .56. For the purposes of measuring variables relevant to the current study, only scales comprising items asking about current adaptability and cohesion were utilized (Adaptability Now and Cohesion Now scales). The Adaptability Now scale measures the current amount of chaos in families. Higher scores are said to indicate more chaos among the family. The Cohesion Now scale measures the degree of separation or connection of family members to the family unit. 
Higher scores on this scale are said to indicate higher cohesion and enmeshment among families.

Family Crisis Oriented Personal Evaluation (F-COPES)

The F-COPES (McCubbin, Olson, \& Larsen 1987) is a 30-item measure that is used to assess how effective the problem-solving coping attitudes and behavior used by families are in response to problems or difficulties. Higher scores on the measure indicate greater use of effective methods of dealing with problems and difficulties. Two components of family interactions are measured: internal family strategies (i.e., problem solving techniques and behaviors that family members use when relating to other members of the nuclear family system) and external family strategies (i.e., behaviors used to acquire help from outside of the family). The F-COPES has a reported internal consistency of .86, and shows good factorial validity and concurrent validity with other measures of family functioning (McCubbin et al., 1987). For the current sample, alpha for the F-COPES was .58.

\section{Parenting Stress Index (PSI)}

The PSI (Abidin, 1995) is a 101-item self-report questionnaire asking individuals to indicate the degree of stress they experience in their role as a parent. Project SAFE uses a 20-item subset of the full PSI. The items utilized in the Project SAFE evaluation produce 2 scales, 1 that assesses parents' appraisal of their competence (Sense of Competence scale) and 1 that assesses the restrictions they experience due to their parental role (Restriction of Role scale). The PSI demonstrates internal consistency (alphas ranging from .70 to .84), test-retest reliability, and validity (Abidin, 1995). A reliability coefficient of $\alpha=.90$ was found for the current sample.

\section{Parent Project Safe Evaluation Form}

The Parent Project SAFE Evaluation Form is an 18-item Likert scale type instrument used to ask nonoffending caregiver participants to rate their satisfaction with their treatment experience. It was specifically constructed for assessing client satisfaction with the Project SAFE program; therefore, no reliability or validity data are currently available. Table 2 contains a description of the items covered by the measure. The Parent Project SAFE Evaluation Form is administered only during the posttreatment assessment. 
Table 2. Mean and Standard Deviations of Items on the Parent Posttreatment Evaluation Form

\begin{tabular}{|c|c|c|}
\hline & $\begin{array}{l}\text { Parents of Symptomatic Children } \\
\qquad(n=31)\end{array}$ & $\begin{array}{l}\text { Parents of Subclinical Children } \\
\qquad(n=24)\end{array}$ \\
\hline Item & $M(S D)$ & $M(S D)$ \\
\hline \multicolumn{3}{|l|}{ Attendance and Environment: } \\
\hline Helpfulness of Weekly Reminders & $2.03(1.38)$ & $2.63(1.53)$ \\
\hline Helpfulness of Child Care & $1.62(1.30)$ & $2.00(1.70)$ \\
\hline Pleasantness of Therapy Rooms & $1.61(0.76)$ & $1.63(0.82)$ \\
\hline \multicolumn{3}{|l|}{ Therapists: } \\
\hline Supportiveness & $1.35(0.61)$ & $1.46(0.66)$ \\
\hline Knowledgeable & $1.52(0.68)$ & $1.50(0.66)$ \\
\hline Prepared & $1.48(0.68)$ & $1.33(0.48)$ \\
\hline \multicolumn{3}{|l|}{ Procedures: } \\
\hline Summary of the Children's Group & $1.60(0.72)$ & $3.22(6.56)$ \\
\hline Meeting with Child Therapist & $1.71(0.82)$ & $1.91(1.00)$ \\
\hline \multicolumn{3}{|l|}{ Session/Program Topics and Goals: } \\
\hline Introduction of Topics & $1.65(0.71)$ & $1.54(0.59)$ \\
\hline Relevance of Topics & $1.77(0.76)$ & $1.63(0.88)$ \\
\hline Extent of Topic Discussion & $1.65(0.61)$ & $1.92(1.02)$ \\
\hline Introduction of Goals & $1.84(0.78)$ & $1.88(0.80)$ \\
\hline Relevance of Weekly Goals & $1.73(0.78)$ & $1.83(0.96)$ \\
\hline Relevance of Overall Goals & $1.58(0.67)$ & $1.58(0.88)$ \\
\hline Satisfaction with Goals Perused & $1.74(0.68)$ & $1.70(0.97)$ \\
\hline \multicolumn{3}{|l|}{ Overall Evaluation of Project SAFE: } \\
\hline Impact on Your Child ${ }^{\mathrm{b}}$ & $2.10(0.94)$ & $2.04(0.86)$ \\
\hline Impact on Yourself $\mathrm{f}^{\mathrm{b}}$ & $2.13(0.96)$ & $2.00(0.93)$ \\
\hline Impact on Your Familyb & $2.16(0.90)$ & $1.96(0.81)$ \\
\hline
\end{tabular}

Note: Parent Project SAFE Evaluation Form items were rated on a scale of 1 (extremely favorable) to 6 (extremely

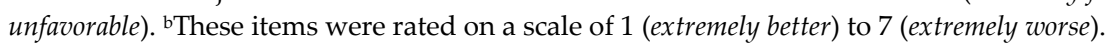

\section{Procedures}

Prior to the pretreatment assessment, all families who were referred to Project SAFE were contacted by the project coordinator and screened based on the inclusion and exclusion criteria. Families were eligible to participate in the study if their child was between 7 and 12 years of age and child protective services investigated the allegation of abuse. If a caregiver of a potential child participant reported that the child currently experienced significantly impaired cognitive/intellectual functioning that would interfere with ability to participate in a group, the child was not eligible to participate in treatment. Eligible parents/ guardians provided informed consent for their child (as well as their own) participation. Children also provided assent to participate in Project SAFE. Caregivers and children participating in Project SAFE completed both pretreatment (typically 1-2 weeks prior to group) and posttreatment (typically 1 week after the completion of group) assessment packets. Caregivers and children were also asked to complete an evaluation of Project SAFE at the posttreatment assessment. Payments of $\$ 20$ were provided to each family at the completion of the pretreatment assessment. This project was approved and conducted in compliance with the University of Nebraska-Lincoln Institutional Review Board. 


\section{Intervention}

Project SAFE is a parallel group treatment program (i.e., child and caregiver groups meet separately but concurrently) that is delivered in 90-minute sessions across 12 weeks. A treatment manual provides content for each weekly session (Hansen et al., 2011). Project SAFE is designed to improve outcomes for children's sense of stigmatization and isolation associated with the abuse, to assist them in exploring and coping with their feelings about the abuse, and to empower them in preventing future victimization. The parallel parent group assists parents in understanding and dealing with their children's behaviors and feelings in an attempt to ensure that the children's in-session therapeutic gains are generalized and maintained. Each Project SAFE session incorporates psychoeducation, skill building, problem solving, and supportive procedures as well as emphasizing strategies to prevent further abuse (Hsu, Sedlar, Flood, \& Hansen, 2002). A manuscript by Tavkar and Hansen (2011) provides a description of the Project SAFE program.

Each Project SAFE group is led by a trained master's level therapist who is currently working toward his or her doctoral degree in clinical psychology. Groups also include cotherapists who are pre-master's-level doctoral students in clinical psychology. Co-therapists receive training by licensed psychologists and experienced master's level therapists prior to delivery of Project SAFE services. The master's level therapist delivers weekly material, facilitates and responds supportively to the group, and engages group members in problem solving and skill building exercises. The role of the cotherapist is to assist in skill modeling, encourage participation, and observe client reaction to treatment (Hansen, Hecht, \& Futa, 1998). All participating therapists receive weekly clinical supervision by licensed psychologists.

\section{Results}

\section{Formation of Groups}

Clinical cutoff scores were used to divide participants into clinically symptomatic and subclinical groups. Measures with established cutoffs (i.e., CDI, CMAS-R, CBCL) were used to form groups in order to increase the clinical utility of the findings. Of the 97 children who received treatment, $64(66 \%)$ presented with clinical symptoms on at least one of the measures with established cutoffs. Of the 64 children who presented with clinical symptoms on at least one of the measures with established cutoffs, 38 (59\%) presented with symptoms above cutoffs on only 1 of the 3 measures used to divide participants (one child presented with symptoms above the cutoff on only the CDI, 5 children presented with symptoms above the cutoff on only the CMAS-R, and 32 children presented with symptoms above the cutoff on only the CBCL). Furthermore, 20 of the 64 (31\%) children presented with clinical symptoms on 2 of the 3 measures used to divide participants (5 children presented with symptoms above the cutoffs on the CDI and the CMAS-R, 4 children presented with symptoms above the cutoffs on the CDI and the CBCL, and 11 children presented with symptoms above the cutoffs on the CMAS-R and the CBCL). Four children presented with clinical symptoms on all 3 of the measures used to divide participants. Finally, 2 children presented with clinical symptoms on at least one measure but also did not complete one of the measures used to divide participants. 


\section{Factors Related to Initial Symptom Presentation}

The groups of clinically symptomatic and subclinical children were compared on demographic variables of caregiver age, caregiver level of education, caregiver marital status, caregiver income, child age, and child gender. Bivariate comparisons revealed no significant differences between the groups on any of the variables. Chi-square tests of independence were used to compare the two groups in terms of characteristics of the sexual abuse the children experienced (see Table 3). Children who initially presented as subclinical were more likely to have experienced abuse perpetrated by a family member when compared to children in the clinically symptomatic group, $\chi^{2}(1)=3.54, p=.047$. No significant differences were found between the two groups with regard to other characteristics of abuse (e.g., penetration, duration of abuse, frequency of abuse). Analysis of variance (ANOVA) was used to compare the groups of symptomatic and subclinical children on measures of family functioning (Table 4). Caregivers of children in the symptomatic group reported significantly less confidence in their competence as parents, $F(1,92)=14.785, p<.001$. No other significant differences between groups on measures of family functioning were found.

Table 3. Percentage of Children in Each Group Experiencing Various Characteristics of Abuse Severity

\begin{tabular}{lcc}
\hline Abuse Characteristics & $\begin{array}{c}\text { Symptomatic Children } \\
(n=62)\end{array}$ & $\begin{array}{c}\text { Subclinical Children } \\
(n=32)\end{array}$ \\
\hline Intrafamilial (\%) & 45.1 & $65.6^{*}$ \\
Involved penetration (\%) & 19.7 & 6.2 \\
Duration was > 1 month (\%) & 60.7 & 58.6 \\
Frequency was $>1$ time (\%) & 60.0 & 62.5 \\
\hline
\end{tabular}

${ }^{*} p<.05$

Table 4. Means and Standard Deviations on Measures of Family Functioning for Each Group

\begin{tabular}{lcc}
\hline & $\begin{array}{c}\text { Symptomatic Children } \\
(n=62)\end{array}$ & $\begin{array}{c}\text { Subclinical Children } \\
(n=35) \\
M(S D)\end{array}$ \\
\hline Cohesion Now on FACES-III & $37.05(6.23)$ & $38.91(5.69)$ \\
Adaptability Now on FACES-III & $24.97(4.29)$ & $23.79(4.08)$ \\
Total Score on the F-COPES & $102.03(15.27)$ & $103.18(15.25)$ \\
Sense of Competence on the PSI & $32.90(6.64)^{*}$ & $27.00(7.83)$ \\
Restriction of Role on the PSI & $20.83(5.33)$ & $18.66(5.63)$ \\
\hline
\end{tabular}

${ }^{*} p<.001$

\section{Treatment Participation}

Of the 97 participants who completed the pretreatment assessment of functioning, 63 participated in the posttreatment assessment and were therefore included in this study's evaluation of Project SAFE treatment outcome and satisfaction. The number of sessions attended by these 63 families ranged from 7 to 12 , and the majority (81\%) completed at least $75 \%$ of the 12 session treatment protocol. There were no differences in terms of the majority of demographic characteristics (i.e., caregiver age, caregiver level of education, 
caregiver marital status, caregiver income, child age) between participants who participated in the posttreatment assessment and participants who did not. Female child participants were more likely than male child participants to participate in the posttreatment assessment, $\chi^{2}(1)=7.10, p=.009$.

\section{Treatment Outcome}

Repeated measure analyses were used to compare the behavioral and psychological functioning of initially symptomatic and subclinical groups at posttreatment (see Table 5). Among children initially in the symptomatic group, all child outcome measures (with the exception of the Internalizing Scale of the CBCL) revealed significant decreases in problematic behavior and distress between pre- and post-treatment. Furthermore, 14 of the 35 $(40 \%)$ children who were initially in the symptomatic group no longer reported clinical symptoms at post-treatment. For children initially categorized in the subclinical group, child report measures of depression (CDI), anxiety (CMAS-R), and posttraumatic stress (CITES-R PTSD Scale) revealed significant decreases between pre- and post-treatment.

In order to explore the possibility of a relationship between dose of treatment and treatment outcome, the number of sessions attended by the participating child was correlated with a change score for each measure that revealed significant decreases between pre- and post-treatment. These analyses were completed separately for children initially in the symptomatic group and for children initially in the subclinical group. Across both the symptomatic and the subclinical group, no significant correlations were found between number of sessions attended by the participating child and pre- to post-treatment change scores on measures that revealed significant decreases across treatment. 
Table 5. Means, Standard Deviations, and One-Way Analyses of Variance (ANOVA) for Child Outcome

\begin{tabular}{|c|c|c|c|c|}
\hline \multirow[b]{2}{*}{ Assessment } & \multirow{2}{*}{$\begin{array}{c}\text { Pre-treatment } \\
M(S D)\end{array}$} & \multirow{2}{*}{$\frac{\text { Post-treatment }}{M(S D)}$} & \multicolumn{2}{|c|}{ ANOVA } \\
\hline & & & $F(d f)^{*}$ & $p \leq$ \\
\hline \multicolumn{5}{|l|}{ Symptomatic Children } \\
\hline $\begin{array}{l}\text { Child Behavior Checklist Externalizing } \\
\text { Subscale }\end{array}$ & $66.80(11.38)$ & $63.27(11.11)$ & $4.98(1,29)$ & .034 \\
\hline $\begin{array}{l}\text { Child Behavior Checklist Internalizing } \\
\text { Subscale }\end{array}$ & $64.93(9.88)$ & $61.90(12.73)$ & $2.61(1,29)$ & .117 \\
\hline Children's Depression Inventory & $57.51(13.30)$ & $50.74(15.75)$ & $7.75(1,34)$ & .009 \\
\hline Children's Loneliness Questionnaire & $39.11(15.40)$ & $29.86(10.89)$ & $13.41(1,34)$ & .001 \\
\hline $\begin{array}{l}\text { Revised Children's Manifest Anxiety } \\
\text { Scale }\end{array}$ & $57.62(12.12)$ & $50.18(13.91)$ & $13.62(1,33)$ & .001 \\
\hline $\begin{array}{l}\text { Children's Fears Related to } \\
\text { Victimization }\end{array}$ & $58.14(11.04)$ & $51.11(10.75)$ & $15.79(1,35)$ & .001 \\
\hline CITES-R PTSD Subscale & $32.06(9.45)$ & $24.83(10.04)$ & $18.05(1,35)$ & .001 \\
\hline CITES-R Social Reactions Subscale & $6.72(6.29)$ & $4.28(3.99)$ & $6.47(1,35)$ & 016 \\
\hline CITES-R Attributions Subscale & $23.33(9.50)$ & $19.11(7.84)$ & $7.86(1,35)$ & .008 \\
\hline \multicolumn{5}{|l|}{ Subclinical Children } \\
\hline $\begin{array}{l}\text { Child Behavior Checklist Externalizing } \\
\text { Subscale }\end{array}$ & $48.63(10.54)$ & $48.75(9.64)$ & $0.01(1,23)$ & .932 \\
\hline $\begin{array}{l}\text { Child Behavior Checklist Internalizing } \\
\text { Subscale }\end{array}$ & $50.83(9.08)$ & $50.17(9.50)$ & $0.13(1,23)$ & .717 \\
\hline Children's Depression Inventory & $44.50(5.97)$ & $40.64(4.17)$ & $9.89(1,27)$ & .004 \\
\hline Children's Loneliness Questionnaire & $25.25(8.08)$ & $23.50(8.29)$ & $1.11(1,27)$ & .302 \\
\hline $\begin{array}{l}\text { Revised Children's Manifest Anxiety } \\
\text { Scale }\end{array}$ & $43.26(10.24)$ & $38.07(10.08)$ & $9.13(1,26)$ & .006 \\
\hline $\begin{array}{l}\text { Children's Fears Related to } \\
\text { Victimization }\end{array}$ & $50.71(9.86)$ & $47.29(10.51)$ & $2.62(1,27)$ & .117 \\
\hline CITES-R PTSD Subscale & $23.82(8.30)$ & $18.14(7.89)$ & $17.29(1,27)$ & .001 \\
\hline CITES-R Social Reactions Subscale & $3.71(4.43)$ & $2.89(2.51)$ & $1.99(1,27)$ & .170 \\
\hline CITES-R Attributions Subscale & $17.04(7.14)$ & $15.71(4.91)$ & $1.84(1,27)$ & .186 \\
\hline
\end{tabular}

${ }^{*} N$ varies because of missing data/measurements.

\section{Client Satisfaction with Group Treatment}

Overall, both children and caregivers rated the program favorably at the post-treatment assessment (Tables 1 and 2, respectively). Caregiver scores indicated satisfaction with the therapeutic environment, therapists, treatment content, and overall impact of therapy. Similarly, child scores indicated satisfaction with group therapists, group topics, and the overall group content. Between-group factorial ANOVAs were performed to examine differences between initially symptomatic and subclinical groups of children on the social validity of Project SAFE. No significant differences were found between groups on mean scores for any of the items, and both groups were equally satisfied with the Project SAFE group treatment program. 


\section{Discussion}

Children who experience CSA are a heterogeneous group, and it is important that treatment programs are capable of addressing a broad range of symptom profiles. Although the majority of the participants in the present study presented with symptoms above the clinical cutoff on at least one measure of behavioral difficulties and emotional distress, 34\% of the participating children in the current study presented with few symptoms following CSA. These findings are consistent with previous research demonstrating that approximately $30 \%$ to $40 \%$ of children who experience CSA will present with little to no symptoms (Putnam, 2003).

While the diagnosis of PTSD is likely the most commonly used label in literature on psychopathology following CSA, it is clear that the experience of sexual abuse can result in symptoms across multiple domains of children's functioning that are not always captured by the PTSD diagnostic criteria (van der Kolk, Roth, Pelcovitz, Sunday, \& Spinazzola, 2005). A robust literature has demonstrated a relationship between child abuse and impairments in areas such as mood and emotion, behavioral control, attachment, and selfconcept (e.g., Cook et al., 2005). Furthermore, many children present with problematic abuse-related cognitions, emotions, and behaviors (e.g., self-blame, shame, sexualized behaviors) that are important targets for treatment but are not necessarily symptoms of a specific psychiatric diagnosis (Deblinger \& Runyon, 2005; Feiring, Simon, \& Cleland, 2009). This study adds to the literature on treatment for CSA by demonstrating that assessing for a broad range of possible outcomes following CSA is important for researchers and clinicians aiming to understand and treat child victims.

Project SAFE is a broad, inclusive, and standardized group treatment for children who have been sexually abused. This study provides evidence that when delivering such an intervention, thorough assessment is critical to ensuring treatment benefits are achieved and appropriate referrals are provided if a time-limited protocol is not sufficient for meeting needs of children and their families. Within this study's group of initially symptomatic children, a great deal of variation existed with regard to the type and number of measures on which children presented with symptoms above clinical cutoffs. This finding is consistent with a large body of literature on discrepancies within informants on ratings of children's social and emotional problems, which demonstrates that there is no single measure for assessing psychopathology in children that provides a definitive way of knowing if children are experiencing specific problems or disorders (e.g., De Los Reyes \& Kazdin, 2005). The broad battery of assessment measures utilized in the Project SAFE protocol helps ensure that children experiencing clinically significant symptoms are identified, that therapists know if treatment has been helpful for reducing symptoms, and that appropriate and informed referrals can be provided if the 12 session protocol is not sufficient for reducing symptoms.

Several findings that add to the CSA treatment research on the heterogeneity within the population of children who experience sexual abuse emerged from the present study. Children who initially presented as subclinical were more likely than symptomatic children to have experienced abuse by a family member. This finding is inconsistent with previous research, which suggests that children abused by a family member are at increased 
risk for psychological difficulties (for a review, see Tyler, 2002). This finding may be due (at least in part) to contextual factors; for instance, children participating in Project SAFE were no longer living in an abusive environment. Furthermore, many of these children had recently witnessed their nonoffending caregiver end a marital or romantic relationship with the perpetrator. This action may have served as a salient indicator of support from the nonoffending caregiver and helped prevent or decrease psychological symptoms (Elliott \& Carnes, 2001).

Caregivers of the children in the current study who presented for treatment with clinically significant symptoms reported higher levels of distress related to their competence as parents when compared to caregivers of children who presented with subclinical symptoms. Caregivers of children who are exhibiting clinical levels of psychological symptoms following CSA likely experience parenting stress both related to the occurrence of CSA and difficulties exhibited by their children. These findings suggest that treatments that provide caregivers with strategies on how to prevent future victimization and improve behavior management skills may increase caregivers' confidence in their ability to care for and monitor the safety of their children (Tavkar \& Hansen, 2011). Furthermore, the distressing emotions and negative cognitions that nonoffending caregivers often experience following sexual abuse can be directly addressed when they are included in treatment. By addressing caregiver's functioning in treatment following CSA, caregivers can leave treatment better equipped to cope independently with the emotional and behavioral reactions of their children (Corcoran, 2004).

The Project SAFE group intervention demonstrated significant improvements in behavioral and psychological functioning in a heterogeneous population of families seeking treatment following CSA. Consistent with the study's hypotheses, both symptomatic and subclinical children demonstrated significant improvements following Project SAFE treatment. These findings provide initial evidence of the efficacy of the Project SAFE treatment and the ability to address the diverse range of symptoms in a group format.

Moreover, all participating children and their caregivers evaluated treatment favorably. This study provides evidence that, regardless of whether or not children displayed high levels of psychiatric symptoms prior to treatment, families viewed the short-term, parallel group treatment as valuable and beneficial. The CAC environment, which is increasingly being used nationally as an access site for services following children's disclosure of abuse, requires broad and inclusive treatments that can be offered promptly and effectively to families in need (Tavkar \& Hansen, 2011). The favorable evidence regarding outcomes and client satisfaction with Project SAFE provided by this study suggests that this manualized protocol could be a helpful new tool for community clinicians working with children and families that experience CSA.

Several limitations to the current study exist, which suggest avenues for future research on the Project SAFE protocol and on CSA in general. Children who receive treatment following CSA represent a subset of the overall population of children who experience CSA, many of whom will never report the abuse to authorities or be supported by an adult in seeking treatment (Kilpatrick, Saunders, \& Smith, 2003). A treatment-seeking population was utilized in the current study, which limits the generalizability of the findings. Future research that includes children who have experienced CSA but do not seek treatment could 
provide broader information about the heterogeneous effects of experiencing CSA. This investigation did not include a follow-up assessment. Future research on the Project SAFE protocol should examine the durability of the effects of the intervention over time and explore for the possibility of emergence of symptoms in initially subclinical children. Finally, this preliminary investigation of Project SAFE did not include a control group. Waitlisttype control groups are difficult and sometimes unethical to create when working with families who experience CSA and are requesting services from helping professionals during the often very stressful time period that follows disclosure by children. However, other researchers conducting treatment efficacy research with families who have experienced CSA have successfully completed randomized trials using a supportive control-treatment (e.g., Deblinger, Stauffer, \& Steer, 2001). Given the preliminary evidence of the efficacy and feasibility of the Project SAFE protocol, this study should be used as a catalyst for evaluations of the treatment using a control condition.

Acknowledgments - We would like to thank all the talented and dedicated therapists who have helped to provide Project SAFE services, especially Elaine K. Martin, who coordinated services from 2009 to 2012.

\section{References}

Abidin, R. R. (1995). The Parenting Stress Index (3rd ed.): Professional manual. Odessa, FL: Psychological Assessment Resources.

Achenbach, T. M. (1991). The Child Behavior Checklist manual. Burlington, VT: University of Vermont.

Achenbach, T. M., \& Rescorla, L. A. (2000). Manual for the ASEBA preschool forms \& profiles. Burlington, VT: University of Vermont, Department of Psychiatry.

Asher, S. R., \& Wheeler, V. A. (1985). Children's loneliness: A comparison of rejected and neglected peer status. Journal of Consulting and Clinical Psychology, 53, 500-505. doi:10.1037/0022-006X.53.4.500

Chaffin, M., \& Shultz, S. (2001). Psychometric evaluation of the Children's Impact of Traumatic Events Scale-Revised. Child Abuse \& Neglect, 25, 401-411. doi:10.1016/S0145-2134(00)00257-X

Cohen, J. A., Mannarino, A. P., \& Deblinger, E. (2006). Treating trauma and traumatic grief in children and adolescents. New York: Guilford.

Cook, A., Spinazzola, J., Lanktree, C., Blaustein, M., Cloite, M., DeRosa, R., ... van der Kolk, B. (2005). Complex trauma in children and adolescents. Psychiatric Annals, 35, 390-398.

Corcoran, J. (2004). Treatment outcome research with the non-offending parents of sexually abused children: A critical review. Journal of Child Sexual Abuse, 13, 59-84. doi:10.1300/J070v13n02_04

Cross, T. P., Jones, L. M., Walsh, W. A., Simone, M., \& Kolko, D. (2007). Child forensic interviewing in children's advocacy centers: Empirical data on a practice model. Child Abuse and Neglect, 21, 1031-1052. doi:10.1016/j.chiabu.2007.04.007

De Los Reyes, A, \& Kazdin, A. E. (2005). Informant discrepancies in the assessment of childhood psychopathology: A critical review, theoretical framework, and recommendations for further study. Psychological Bulletin, 4, 483-509. doi:10.1037/0033-2909.131.4.483

De Luca, R. V., Boyes, D. A., Furer, P., Grayston, A. D., Hiebert-Murphy, D. (1992). Group treatment for child sexual abuse. Canadian Psychology, 33, 168-179. doi:10.1037/h0078711

Deblinger, E., \& Runyon, M. K. (2005). Understanding and treating feelings of shame in children who have experienced maltreatment. Child Maltreatment, 10, 364-376. doi:10.1177/1077559505279306 
Deblinger, E., Stauffer, L. B., \& Steer, R. (2001). Comparative efficacies of supportive and cognitive behavioral group therapies for young children who have been sexually abused and their nonoffending mothers. Child Maltreatment, 6 ,332-343. doi:10.1177/1077559501006004006

Elliott, A. N., \& Carnes, C. N. (2001). Reactions of nonoffending parents to the sexual abuse of their child: A review of the literature. Child Maltreatment, 6 , 314-331. doi:10.1177/1077559501006004005

Feindler, E., Rathus, J., \& Silver, L. (2003). Assessment of family violence: A handbook for research and practitioners. Washington, DC: American Psychological Association. doi:10.1037/10462-000

Feiring, C., Simon, V. A., \& Cleland, C. M. (2009). Child sexual abuse, stigmatization, internalizing symptoms, and the development of sexual difficulties and dating aggression. Journal of Consulting and Clinical Psychology, 77, 127-137. doi:10.1037/a0013475

Finkelhor, D., \& Berliner, L. (1995). Research on the treatment of sexually abused children: A review and recommendations. Journal of the American Academy of Child and Adolescent Psychiatry, 34, 14081423. doi:10.1097/00004583-199511000-00007

Hansen, D. J., Futa, K. T., Hecht, D. B., Sedlar, G., Yancey, C. T., Cronch, L. E., . . Friedenberg, S. (2011). Project SAFE group intervention treatment manual. Lincoln, NE: University of NebraskaLincoln, Department of Psychology.

Hansen, D. J., Hecht, D. B., \& Futa, K. T. (1998). Child sexual abuse. In V. B. van Hassselt \& M. Hersen (Eds.), Handbook of psychological treatment protocols for children and adolescents (pp. 153-178). Mahwah, NJ: Erlbaum.

Harvey, S. T., \& Taylor, J. E. (2010). A meta-analysis of the effects of psychotherapy with sexually abused children and adolescents. Clinical Psychology Review, 30, 517-535. doi:10.1016/j.cpr.2010.03.006

Hetzel-Riggin, M. D., Brausch, A. M., \& Montgomery, B. S. (2007). A metaanalytic investigation of therapy modality outcomes for sexually abused children and adolescents: A exploratory study. Child Abuse \& Neglect, 31, 125-141. doi:10.1016/j.chiabu.2006.10.007

Hsu, E. (2003). Parallel group treatment for sexually abused children and their nonoffending parents: An examination of treatment integrity and child and family outcome and satisfaction. Unpublished doctoral dissertation, University of Nebraska-Lincoln, Lincoln, NE.

Hsu, E., Sedlar, G., Flood, M. F., \& Hansen, D. J. (2002). Child sexual abuse. In M. Hersen (Ed.), Clinical behavior therapy: Adults and children (pp. 449-473). New York, NY: Wiley.

Hubel, G. S., Maldonado, R. C., Tavkar, P., Hansen, D. J., \& Flood, M. F. (2011). Group treatment for a sexually abused child and a non-offending caregiver: Case study and discussion. Clinical Case Studies, 10, 360-375. doi:10.1177/1534650111422376

Jackson, S. L. (2004). A USA national survey of program services provided by child advocacy centers. Child Abuse \& Neglect, 28, 411-421. doi:10.1016/j.chiabu.2003.09.020

Kilpatrick, D., Saunders, B., \& Smith, D. (2003). Youth victimization: Prevalence and implications. Washington, DC: U.S. Department of Justice, National Institute of Justice report.

Kovacs, M. (1992). Children's Depression Inventory. North Tonawanda, NY: Multi Health Systems.

Lang, J. M., Ford, J. D., \& Fitzgerald, M. M. (2010). An algorithm for determining use of traumafocused cognitive behavioral therapy. Psychotherapy: Theory, Research, Practice, Training, 47, 554569. doi:10.1037/a0021184

McCubbin, H. I., Olson, D. H., \& Larsen, A. S. (1987). F-COPES: Family Crisis Oriented Personal Evaluation Scales. In H. I. McCubbin \& A. I. Thompson (Eds.), Family assessment inventories for research and practice (pp. 259-270). Madison, WI: University of Wisconsin.

National Children's Alliance. (2009). Ending child abuse and neglect after disclosure. Retrieved from http://www.nationalchildrensalliance.org/index.php?s24 
Ollendick, T. H. (1983). Reliability and validity of the Revised Fear Survey Schedule for Children (FSSC-R). Behaviour Research and Therapy, 21, 685-692. doi:10.1016/0005-7967(83)90087-6

Olson, D. H. (1986). Circumplex Model VII: Validation studies and FACES-III. Family Process, 25, 337350. doi:10.1111/j.1545-5300.1986.00337.x

Prinstein, M. J., \& La Greca, A. M. (2002). Peer crowd affiliation and internalizing distress in childhood and adolescence: A longitudinal follow-back study. Journal of Research on Adolescence, 12, 325-351. doi:10.1111/1532-7795.00036

Putnam, F. W. (2003). Ten-year research update review: Child sexual abuse. Journal of the American Academy of Child \& Adolescent Psychiatry, 42, 269-278. doi:10.1097/00004583-200303000-00006

Reynolds, C. R. (1980). Concurrent validity of What I Think and Feel: The Revised Children's Manifest Anxiety Scale. Journal of Consulting and Clinical Psychology, 48, 774-775. doi:10.1037/0022006X.48.6.774

Reynolds, C. R., \& Richmond, B. O. (1985). Revised Children's Manifest Anxiety Scale Manual. Los Angeles: Western Psychological Services.

Saunders, B. E., Berliner, L., \& Hanson, R. F. (Eds.). (2004). Child physical and sexual abuse: Guidelines for treatment. Charleston, SC: National Crime Victims Research and Treatment Center.

Sawyer, G. K., Yancey, C. T., Hsu Tsao, E., Wynne, A, Hansen, D. J., \& Flood, M. F. (2005, November). Parallel group treatments for sexually abused youth and their nonoffending parents: Treatment integrity, outcomes and social validity of Project SAFE. Poster presented at the Association for Behavioral and Cognitive Therapies, Washington, DC.

Saywitz, K. J., Mannarino, A. P., Berliner, L., \& Cohen, J. A. (2000). Treatment for sexually abused children and adolescents. American Psychologist, 55, 1040-1049. doi:10.1037/0003-066X.55.9.1040

Storch, E. A., Brassard, M. R., \& Masia-Warner, C. L. (2003). The relationship of peer victimization to social anxiety and loneliness in adolescence. Child Study Journal, 33, 1-18.

Tavkar, P., \& Hansen, D. J. (2011). Interventions for families victimized by child sexual abuse: Clinical issues and approaches for child advocacy center-based services. Aggression and Violent Behavior, 16 , 188-199. doi:10.1016/ j.avb.2011.02.005

Tyler, K. A. (2002). Social and emotional outcomes of childhood sexual abuse: A review of recent research. Aggression and Violent Behavior, 7, 567-587. doi:10.1016/S1359-1789(01)00047-7

van der Kolk, B. A., Roth, S., Pelcovitz, D., Sunday, S., \& Spinazzola, J. (2005). Disorders of extreme stress: The empirical foundation of a complex adaptation to trauma. Journal of Traumatic Stress, 18, 389-399. doi:10.1002/jts.20047

Wolfe, V. V. (2006). Child sexual abuse. In E. J. Mash \& R. A. Barkley (Eds.), Treatment of childhood disorders (pp. 647-727). New York, NY: Guilford Press.

Wolfe, V. V., Gentile, C., Michienzi, T., Sas, L., \& Wolfe, D. A. (1991). The children's impact of traumatic events scale: A measure of post-sexual-abuse PTSD symptoms. Behavioral Assessment, 13, 359-383.

Wolfe, V. V., \& Wolfe, D. A. (1986). The Sexual Abuse Fear Evaluation (SAFE): A subscale for the Fear Survey Schedule for Children-Revised. Unpublished questionnaire. Children's Hospital of Western Ontario, London, Ontario. 


\section{Author Notes}

Grace S. Hubel, MA, is a doctoral student in the clinical psychology training program at the University of Nebraska-Lincoln and a psychology intern at the Medical University of South Carolina. Her research interests include outcome and dissemination research with evidence-based treatments for maltreated children and children with behavioral difficulties.

Christopher Campbell, PhD, is a postdoctoral fellow at the University of Oklahoma Health Sciences Center. He completed his doctoral training in the clinical psychology training program at University of Nebraska-Lincoln. His primary clinical and research interests include the assessment and treatment of youth who have experienced child maltreatment, youth with problematic sexual behavior, and youth with problematic parent-child and/or teacher-child relationships.

Tiffany West, MA, is a doctoral student in the clinical psychology training program at University of Nebraska-Lincoln. Her primary research and clinical interests are in child maltreatment, specifically childhood sexual abuse.

Samantha Friedenberg, MA, is a doctoral student in the clinical psychology training program at University of Nebraska-Lincoln. Her research interests have broadly spanned the psychological effects of child sexual abuse, and she is currently investigating the phenomenon of sexual revictimization within childhood and adolescence.

Alayna Schreier, MA, is a doctoral student in the clinical psychology training program at University of Nebraska-Lincoln. Her primary clinical and research interests are in child maltreatment, specifically the development and dissemination of effective treatment interventions for children and their families.

Mary Fran Flood, PhD, is director of the University of Nebraska-Lincoln Psychological Consultation Center. Her research and clinical interests focus on child maltreatment, assessment and intervention with young children and their families, and family interaction approaches for treatment of children. She has published research articles and chapters on child protection, school-based services, and ecological and policy issues affecting children and families.

David J. Hansen, PhD, is director of clinical training and professor of the Psychology Department at University of Nebraska-Lincoln. He directs and supervises all Project SAFE services. Dr. Hansen has published extensively on maltreatment, clinical assessment and intervention, and child and adolescent social competence and adjustment, among other topics. 